\title{
Normas de associação semântica para 20 categorias em adultos e idosos
}

\author{
Suelen Bordignon - Universidade Federal do Rio Grande do Sul, Porto Alegre, Brasil \\ Murilo R.Zibetti - Universidade Federal do Rio Grande do Sul, Porto Alegre, Brasil \\ Clarissa M. Trentini - Universidade Federal do Rio Grande do Sul, Porto Alegre, Brasil \\ Ana Cristina Resende - Universidade Católica de Goiás, Goiânia, Brasil \\ Carla A. S. M. Minervino - Universidade Federal da Paraíba \\ José Humberto da Silva-Filho - Universidade Federal do Amazonas, Manaus, Brasil \\ Josiane Pawlowski - Universidade Federal do Rio de Janeiro, Rio de Janeiro, Brasil \\ Maycoln L. M. Teodoro - Universidade Federal de Minas Gerais, Belo Horizonte, Brasil \\ Neander Abreu - Universidade Federal da Babia, Salvador, Brasil
}

\begin{abstract}
Resumo
O desenvolvimento de normas para categorias semânticas é necessário para a compreensão do modo como os indivíduos organizam o conhecimento na memória e para a construção de instrumentos de avaliação cognitiva. O objetivo do presente estudo é fornecer normas associativas de 20 categorias semânticas para adultos e idosos brasileiros. Participaram do estudo 236 pessoas, entre 40 e 88 anos, das cinco regiões brasileiras. Os participantes responderam a um questionário de dados sociodemográficos e a um questionário de itens para categorias semânticas, e foram solicitados a produzir os cinco primeiros exemplares de cada categoria apresentada. Os resultados foram agrupados e são apresentadas as normas das categorias (exemplares, tamanho da categoria, respostas corretas, em branco e erros), bem como as respostas de cada categoria que foram comuns a todas as regiões brasileiras.

Palavras-chave: semântica; normas; associação; adulto; idoso.
\end{abstract}

Semantic association norms for 20 categories in adults and elderly

\begin{abstract}
The development of semantic category norms is necessary to assist in the understanding of how individuals organize knowledge in memory, and to the creation of cognitive assessment instruments. The aim of this study is to provide associative norms of 20 semantic categories for Brazilian adults and elderly. The study included 236 participants from 40 to 88 years of age from the five Brazilian regions. They answered a socio-demographic questionnaire and a semantic category questionnaire, in which they were asked to produce the first five items of each category. Results are presented in category norms (items, category set size, correct responses, no responses and errors), as well as the responses that were common to all Brazilian regions belonging to each category.

Keywords: semantics; standards; association; adult; aged.
\end{abstract}

Normas de asociación semántica para 20 categorías en adultos y ancianos

\begin{abstract}
Resumen
Es necesario el desarrollo de normas para categorías semánticas para poder comprender cómo las personas organizan el conocimiento en la memoria y para la construcción de instrumentos de evaluación cognitiva. El objetivo de este estudio es proporcionar normas asociativas de 20 categorías semánticas para muestras de brasileños adultos y ancianos. Participaron 236 personas entre 40 y 88 años, de cinco regiones de Brasil. Los participantes completaron un cuestionario de datos sociodemográfico y un cuestionario de ítems para categorías semánticas y se les solicitó la producción de los cinco primeros ejemplares de cada categoría presentada. Los resultados fueron agrupados y son presentadas las normas de las categorías (ejemplares, tamaño de categoría, respuestas correctas, en blanco y errores), así como las respuestas de cada categoría, comunes a todas las regiones de Brasil. Palabras-clave: semántica; normas; asociación; adulto; anciano.
\end{abstract}

\section{Introdução}

A disponibilidade de bancos de normas de palavras e imagens tem auxiliado pesquisadores no desenvolvimento de estudos em Psicologia e áreas correlatas. A utilização de estímulos normatizados reduz os efeitos indesejáveis de confusão dos experimentos, contribuindo para a compreensão teórica de fenômeno ou para desenhos experimentais. Como exemplo do uso de normas, pode-se citar que, quando a familiaridade de um determinado estímulo não é controlada em um teste de memória, isso pode repercutir na facilitação da 
tarefa para um grupo experimental que apresenta maior contato com os estímulos (familiaridade), enviesando as conclusões do estudo. Dessa forma, a criação de bancos de normas, busca minimizar esse tipo de efeito, além de ser fonte, por si só, de conhecimento.

$\mathrm{Na}$ literatura, destacam-se as normas internacionais de frequência de palavras de Kucera e Francis (1967), de categorização semântica de Battig e Montague (1969), de familiaridade e tipicidade de Hampton e Gardiner (1983), e de concretude de Paivio, Yuille e Madigan (1968). Em se tratando de imagens, cita-se o estudo de Snodgrass e Vanderwart (1980), que apresentam dados de concordância de nomeação, imageabilidade, familiaridade e complexidade visual.

No Brasil, o desenvolvimento de normas de palavras foi realizado principalmente na última década e compreende, na maioria das vezes, o estudo da frequência com que certas respostas são emitidas. Destaca-se a criação de um banco de dados de frequências de palavras na fala e na escrita de Sardinha (2000), os estudos de associação semântica, concretude, frequência e emocionalidade de Stein e Gomes (2009), as pesquisas sobre categorização semântica em adultos de Janczura (1996) e em idosos de Van Erven (2010), de concretude de palavras de Janczura, Castilho, Rocha e Van Erven (2007), de associação de palavras completando sentenças de Janczura (2005) e os estudos de Salles sobre associação semântica livre de palavras em universitários (Salles et al., 2008) e em adultos jovens e idosos (Zortea \& Salles, 2012).

A criação de normas é útil ao entendimento de como os indivíduos organizam o conhecimento, além de servir de fonte no desenvolvimento de tarefas e instrumentos de mensuração (Buschke, 1984; Kaplan, Goodglass, \& Weintraub, 2001). Ao que diz respeito, especificamente, à categorização semântica, pesquisadores buscam compreender o modo como os seres humanos organizam e relacionam mentalmente as informações. O banco de normas de categorização semântica mais difundido na literatura é o de Battig e Montague (1969), o qual atualizou e expandiu os primeiros estudos com 43 categorias verbais de Cohen, Bousfield e Whitmarsh (1957).

Ao realizar a coleta de dados, Battig e Montague (1969) apresentavam um caderno com 56 categorias (uma por página), para as quais os participantes escreviam o maior número de exemplares possíveis no período de 30 segundos. O estudo foi realizado com universitários de duas instituições (Universidades de Illinois e de Maryland). A motivação para a construção de normas foi a ascensão de estudos sobre aprendizagem e organização do conhecimento, principalmente, utilizando recordação livre.

No Brasil, citam-se os estudos de Jancuza e de Van Erven na construção de normas de categorias semânticas. Em 1996, Janczura apresentou os dados de associação semântica para 69 categorias. Solicitava aos participantes a produção do primeiro exemplar de cada categoria. Foram disponibilizadas informações, como tamanho das categorias, número de acertos e respostas em branco, exemplares e sua frequência de aparição. Para garantir maior fidedignidade ao estudo, realizou randomização com cinco formas de aplicação. $\mathrm{O}$ estudo foi realizado com 220 universitários de Brasília, sendo que, na amostra, havia participantes provenientes de outros estados brasileiros (Janczura, 1996). Em uma pesquisa em desenvolvimento, normas associativas de categorias em idosos vem sendo criadas por Van Erven e Janczura (2007). Para tanto, em estudo prévio, foram selecionadas 47 categorias do estudo inicial de Janczura (1996), também, com o intuito de comparar o modo como crianças, jovens e idosos produzem exemplares e categorizam.

Dentre os estudos revisados, nota-se a existência de formas variadas de coleta de dados, que incluem o método de primeira resposta e de respostas múltiplas. Janczura (1996) e Van Erven (2010) se utilizaram de uma única palavra associada à categoria; Stein, Feix e Rohenkohl (2006) solicitaram aos participantes que produzissem três exemplares; Battig e Montague (1969) disponibilizaram 30 segundos para que os participantes produzissem o número de exemplares possíveis; Yoon et al. (2004) solicitaram cinco exemplares para cada categoria. A discussão sobre a melhor forma de coleta está relacionada aos processos cognitivos subjacentes a cada uma delas.

Em 1982, McEvoy e Nelson realizaram um estudo para avaliar se as formas de coleta para um exemplar ou mais de um diferiam nos resultados de associação semântica obtidos. Eles coletaram normas para 106 categorias semânticas e as compararam com as normas já existentes. Como resposta, obtiveram grandes similaridades entre os resultados, porém o fato de não serem idênticas foi compreendido como sendo processos subjacentes distintos. Os críticos, quanto ao método de respostas múltiplas, sugerem que é possível que haja um encadeamento de respostas, ou seja, ao emitir um exemplar, o próximo poderia estar associado a esse e não mais à categoria. Além disso, é possível que ao serem solicitados a produzir 
um número de exemplares, possam evocar, simultaneamente, um conjunto de respostas relacionadas entre si (Nelson, McEvoy, \& Bajo, 1988).

No Brasil, existe uma demanda crescente por instrumentos de avaliação neuropsicológica e, embora haja disponibilidade de acesso a testes internacionais, sempre se considera temerária a transposição pura e simples dos dados normativos, sobretudo quando os instrumentos envolvem o uso da linguagem. A construção de normas de palavras e imagens, no âmbito da avaliação psicológica e neuropsicológica, apresenta relevância na construção de instrumentos, visando à possibilidade de escolha de itens adequados que condigam com o objetivo da mensuração e no controle de variáveis intervenientes. No que diz respeito à avaliação da memória por meio de pistas semânticas, a existência de normas de frequência propicia aos pesquisadores maior poder de decisão e manipulação das variáveis, tendo em vista que há facilitação da recuperação de itens que apresentam maior força de associação (Koriat, 2000).

A frequência de produção de exemplares de categorias tem sido associada fortemente a construtos como tipicidade e acessibilidade conceitual. Esses dados demonstram que os exemplares mais frequentes apresentados nas categorias são os itens mais típicos ou mais protuberantes dentro das categorias, com maior facilidade e rapidez de identificação de pertencimento à categoria (Janczura \& Nelson, 2006).

Esse artigo tem como objetivo apresentar a criação de normas para 20 categorias semânticas. Futuramente, pretende subsidiar o processo de construção de um instrumento de avaliação de memória e aprendizagem visoverbal por meio de estímulos desenhados. $\mathrm{O}$ desenvolvimento de normas para categorias semânticas se fez necessário, tendo em vista limitações dos bancos de dados disponíveis, no que diz respeito a fornecer estímulos necessários à construção do instrumento. Dentre as 69 categorias normatizadas de Janczura (1996), apenas 11 delas poderiam ser utilizadas, visto que, em algumas, observou-se conotação emocional (ex.: artigo religioso), sobreposição de categorias (ex.: animal de fazenda e animal selvagem), dificuldade para representar de forma pictórica (ex.: elemento químico) e categorias abstratas (ex.: emoção). Os mesmos impedimentos são reafirmados para o estudo de normas com idosos (Van Erven, 2007), que fizeram uso de 43 das 69 categorias utilizadas por Janczura (1966).

Considerando o caráter essencialmente cultural, dinâmico e semiótico da linguagem, além das limitações já citadas para que fosse possível a utilização de normas já existentes, destaca-se a necessidade da coleta de dados em todas as cinco regiões brasileiras, visando contemplar as diversidades e peculiaridades de cada uma delas. $\mathrm{O}$ uso de instrumentos que propõem avaliar e orientar acerca de possíveis alterações ou deterioração de funções cognitivas demanda um empenho na produção de dados normativos que considerem a diversidade cultural e diferentes níveis de acesso à escolarização em pessoas de grupos não clínicos. Considerou-se, também, a idade dos participantes como importante variável. $\mathrm{Na}$ realização deste estudo, buscou-se a participação de pessoas com mais de 40 anos.

Dessa forma, o objetivo deste estudo foi fornecer normas brasileiras de associação semântica para 20 categorias, apresentar a frequência absoluta dos conceitos, o número de conceitos apresentados para cada categoria, o número de erros e respostas em branco, levando em consideração duas apresentações: somente o primeiro conceito e com os cinco exemplares solicitados. Posteriormente, são apresentados os conceitos que foram comuns a todas as regiões dentro da categoria, ou seja, ao menos tiveram frequência igual a um, em cada uma das cinco regiões brasileiras.

\section{Método}

\section{Participantes}

Participaram do estudo 236 pessoas entre 40 e 88 anos, sendo que 160 eram do sexo feminino $(67,8 \%)$ e 76, do sexo masculino. A seleção da amostra foi realizada por meio de estratificação da população brasileira, levando em consideração os dados do Censo de 2010 (IBGE, 2011) para distribuição de habitantes por faixas etárias (40-49 anos, 50-59 anos, 60-69 anos e 70-79 anos, 80 anos ou mais) em cada uma das cinco regiões. Os participantes foram convidados por conveniência do pesquisador, após preencher os critérios acima citados.

A amostra estudada contou com 20 respondentes da região Centro-Oeste, 18 da região Norte, 46 da região Sul, 94 respondentes do Sudeste e 58 da região Nordeste. Como critério de inclusão, os participantes deveriam ter nascido e morar na região no momento da coleta.

Dentre os participantes que preencheram todos os dados do questionário $(90,2 \%)$, a maioria $(27,2 \%)$ pertence à classe econômica $\mathrm{B} 2$, seguida pelas classes $\mathrm{B} 1$ (26,3\%), C1 (15,5\%), A2 (15\%), C2 (8,9\%), D (4,7\%) e A1 (2,3\%). Em relação à escolaridade, a maior parte dos respondentes $(23,72 \%)$ apresentava ensino médio 
completo; $20,3 \%$ ensino fundamental incompleto; $15,7 \%$ ensino superior completo; $14,4 \%$ pós-graduação; $12,7 \%$ ensino superior incompleto; $5,9 \%$ ensino fundamental completo; $5,5 \%$ ensino médio incompleto e 1,3\% não apresentavam nenhuma escolaridade.

\section{Instrumentos}

Questionário de dados sociodemográficos: objetivou obter informações a respeito dos participantes, tais como idade, sexo, escolaridade, local de nascimento e classe econômica.

Questionário de itens para categorias semânticas: desenvolvido para fins desta pesquisa, é composto por 20 categorias semânticas (frutas, animais, móveis, joias, ferramentas de carpinteiro, meios de comunicação, artigos escolares, peças de vestuário, instrumentos musicais, utensílios de cozinha, instrumentos de limpeza, meios de transporte, coisas que servem para iluminar, artigos de higiene pessoal, objetos usados pela costureira, objetos associados ao esporte, doces, brinquedos, partes do corpo e coisas de bebê). Das 20 categorias estudadas, 10 foram extraídas dos estudos de Battig e Montague (1969), quatro delas do estudo de Petersen et al. (1992) e seis foram criadas pelo Núcleo de Estudos em Avaliação Psicológica e Psicopatologia (NEAPP) da UFRGS. Ao responderem o questionário, os participantes receberam a seguinte instrução "vou apresentar a você diferentes categorias de palavras e vou solicitar que você escreva as cinco primeiras palavras concretas que lhe vierem à cabeça e que digam respeito àquela categoria", seguida por dois exemplos. A forma de responder aos questionários (verbal ou escrita) foi registrada, mas não controlada nesse estudo. Quando respondido de forma verbal, o avaliador realizava o registro das respostas na planilha.

\section{Procedimentos}

Para realização do presente estudo, professores de sete universidades das regiões brasileiras foram contatados (PUC-Goiás, UFAM, UFBA, UFMG, UFP, UFRJ e USF). Na região Sul, os três primeiros autores do presente artigo foram responsáveis pela coleta. Por meio de uma pesquisa de levantamento (Robson, 1993), os participantes responderam de forma individual a um questionário de dados sociodemográficos e ao questionário de itens por categoria semântica. As coletas foram realizadas nos estados de Goiás, Bahia, Paraíba, Amazonas, Minas Gerais, São Paulo, Rio de Janeiro e Rio Grande do Sul. Todos os procedimentos éticos em relação a trabalhos científicos foram atendidos.
Os procedimentos de pesquisa adotados foram os mesmos, independentemente da forma de resposta (verbal ou escrita). O tempo para responder aos questionários era livre. Os dados foram digitados tal qual apareciam nos protocolos. Palavras incomuns para os digitadores eram verificadas em dicionário e em sites de busca para avaliar sua existência. A digitação dos dados foi adequada às normas culta da língua. Análises de frequência foram realizadas no programa estatístico SPSS 17.

Algumas adequações foram feitas em relação à categorização das respostas. Palavras com plural foram transformadas em singular (ex.: a palavra "mesas" foi substituída por "mesa") e substantivos de gênero regular foram agrupados. Grupos de palavras e frases com frequência igual a um que apresentavam equivalência semântica foram unidos (ex.: “jogo de sofás" foi considerado como um item pertencente a "sofá", na categoria móveis). Palavras estrangeiras, quando apropriado, foram traduzidas ao português (ex.: "shampoo" foi reescrito "xampu"), ou mantidas no formato apresentado (ex: e-mail). Foram considerados como sendo o mesmo conceito, as palavras sinônimas (ex.: "tangerina", "mexerica" e "bergamota") e mantido aquele que apresentasse maior frequência (nesse caso, "bergamota"). Quando as respostas representavam marcas comerciais, foram substituídas pela sua denominação, mesmo quando sua frequência fosse mais representativa (ex.: "Bombril' foi denominada "palha de aço"). Gêneros irregulares não sofreram mudanças, e ambos permaneceram no banco de dados (ex.: "boi" e "vaca"). Itens com frequência igual a um foram agrupados em categorias superiores, quando estas existiam (ex.: "coruja" que apresentava frequência igual a um foi agrupada em "ave"). Esses critérios de adequação de respostas em conceitos únicos foi baseado em Janczura (1996).

\section{Resultados}

Para a avaliação das respostas, três juízes, de forma conjunta, foram solicitados a julgar as respostas em: a) resposta relacionada à categoria, b) erro ou c) resposta em branco. Como resultado, obteve-se para a produção dos cinco itens: $96,2 \%$ das respostas corretas (ou seja, respostas pertencentes às categorias); $1,8 \%$ foram consideradas erros e $2 \%$ representaram respostas em branco. Seguindo o mesmo critério, quando considerada somente a primeira resposta, obteve-se: $99 \%$ das respostas corretas; $0,82 \%$ consideradas erros e $0,21 \%$ respostas em branco. 
A apresentação dos resultados segue padronização dos estudos de Janczura (1996), que apresenta tamanho da categoria $(\mathrm{T})$, ou seja, a quantidade de diferentes exemplares emitidos como resposta; número de respostas corretas $(\mathrm{C})$; respostas em branco (B); número de erros (E) e frequência absoluta das respostas ao lado de cada exemplar. O presente trabalho foi realizado por meio de coleta de respostas múltiplas, diferindo do estudo anterior, que se utilizou da coleta de um exemplar para cada categoria. São apresentados dois conjuntos de normas para cada categoria: o primeiro lista a frequência de aparição dos conceitos, levando-se em consideração as cinco respostas emitidas pelos participantes (Tabela 1); e o segundo bloco apresenta as normas do primeiro conceito emitido pelos participantes a cada uma das categorias, em ordem de frequência (Tabela 2). A Tabela 1 e a Tabela 2 apresentam os dez conceitos mais frequentes para cada categoria. As normas completas, com todos os conceitos por categoria podem ser obtidas por meio do e-mail da primeira autora (bordignon.suelen@gmail.com).

São apresentados, na Tabela 3, os três conceitos mais frequentes em todas as regiões brasileiras. A tabela apresenta o percentual de cada um dos conceitos dentro da categoria (ex.: dentre todas as frutas citadas pela região Nordeste, o exemplar "banana" representa $11,4 \%$ ). Da mesma forma, as normas completas de todos os conceitos que tiveram frequência igual ou superior a um em todas as regiões e as frequências absolutas de aparição de cada um dos conceitos por região são disponibilizadas por meio da solicitação a primeira autora (bordignon.suelen@gmail.com).

\section{Discussão}

Além de ser uma fonte de conhecimento sobre o modo como as pessoas organizam as informações categoricamente, as normas aqui apresentadas podem servir de base para a criação de tarefas de avaliação cognitiva. Uma das motivações para o desenvolvimento dessas normas foi a construção de itens para um instrumento visoverbal de avaliação de memória e aprendizagem, que se utilizará de pistas categóricas. A construção desse instrumento deverá ser apresentada em estudos futuros, e as presentes normas auxiliarão não somente na criação desse instrumento como de outros.

$\mathrm{O}$ artigo fornece normas associativas para 20 categorias semânticas para a população brasileira acima de 40 anos e apresenta dados de frequências absolutas dos exemplares, tamanho da categoria, número de respostas corretas, incorretas e em branco. Disponibiliza, também, os conceitos relativos à análise com as cinco respostas produzidas e, somente, com o primeiro item produzido em cada categoria. Os conceitos comuns às cinco regiões brasileiras de cada categoria são destacados.

As normas disponibilizadas neste estudo apresentam algumas diferenças em relação àquelas já acessíveis na literatura brasileira Janczura, 1996; Van Erven, 2007). A seleção das categorias para normatização neste trabalho levou em consideração a maior probabilidade de produção de exemplares que pudessem ser desenhados posteriormente, que apresentassem reduzida conotação emocional e a busca pela não sobreposição de categorias. Buscava, também, coletar dados em todas as cinco regiões brasileiras, respeitando a diversidade dos aspectos culturais. A idade dos participantes foi considerada, fazendo parte da amostra pessoas acima de 40 anos.

Em relação às categorias coletadas, identifica-se que doces foi a que mais produziu conceitos que, possivelmente, apresente maiores dificuldades para serem desenhados ou identificados. Em relação à sobreposição de categorias, as normas coletadas apresentaram conceitos comuns a mais de uma categoria (ex.: bicicleta e carro como meio de transporte e brinquedo; barco em meio de transporte e objetos associados ao esporte). Essa sobreposição de itens em categorias distintas, também, ocorre no estudo de Battig e Montague (1969), Janczura (1996) e Van Erven (2007), e em função de determinados conceitos pertencerem a diversas categorias semânticas. No uso desse banco de dados, destaca-se a necessidade de avaliar os conceitos que pertencem a mais de uma categoria na construção de instrumentos e tarefas que não tenham essa intenção. Conforme se infere da revisão teórica, bem como de constatação empírica, confirmou-se que existem itens que são mais adequados e outros menos, seja pela estabilidade ou multiplicidade de nomes.

O presente estudo fez uso da geração de cinco exemplares para cada categoria, utilizado por vezes, por outros pesquisadores em normas de associação (Yoon et al., 2004). Tendo em vista que estudos nacionais consideram o primeiro exemplar na geração de normas, há limitações para a realização de comparações entre eles. É notável, no entanto, que a ordem de frequência dos conceitos, na análise das cinco respostas e das primeiras, foi similar. Apesar de serem conhecidas algumas das limitações quanto à solicitação de cinco exemplares, ao invés de um, para cada categoria (Nelson, McEvoy, 
Tabela 1. Normas de Categorias Semânticas de acordo com as Cinco Respostas Emitidas

Animais (T: 81, C: 1177, E: 0, B: 3): cachorro 203, gato 173, cavalo 116, vaca 55, leão 51, pássaro 48, macaco 38, boi 36, coelho 35, elefante, galinha 33, (...)

Artigos escolares (T: 42, C: 1164, E: 6, B: 10): lápis 216, caderno 206, borracha 167, caneta 166, livro 119 , régua 73 , apontador 41 , mochila 27 , estojo 17 , papel $16,(\ldots)$

Artigos de higiene pessoal (T: 41, C: 1130, E: 18, B: 32): sabonete 213, xampu 148, pasta de dente 140, desodorante 81 , escova de dente 69 , creme 50 , escova 42 , perfume, toalha 39 , papel higiênico $36,(.$.

Brinquedos (T: 92, C: 1120, E: 20, B: 39): boneca 196, carrinho 163, bola 157, bicicleta 62, quebra-cabeça 45, pião, vídeo-game 38, corda 29, jogo, velotrol 26, (...)

Coisas de bebê (T: 74, C: 1144, E: 25, B: 10): fralda 184, chupeta 172, mamadeira 146, berço 82, chocalho, sapatinho 44, carrinho 38 , talco 34 , babador 29 , macacão $28,(.$.

Coisas que servem para iluminar (T: 31, C: 1069, E: 40, B: 71): lâmpada 218, lanterna 191, vela 166, lampião 63, farol 60, abajur 58, lamparina 43, sol 37, fósforo 30, luminária 27, (...)

Doces (T: 109, C: 1152, E: 10, B: 16): bolo 86, brigadeiro 80, de leite 79, pudim 76, de goiaba 72, chocolate 49 , cocada 47 , pé de moleque, torta 44 , sorvete $35,(.$.

Ferramentas de carpinteiro (T: 63, C: 1048, E: 65, B: 64): martelo 192, serrote 150, prego 70, plaina 59, alicate 56 , chave de fenda 48 , formão 43 , serra 37 , lixa 36 , furadeira $35,(. .$.

Frutas (T: 52, C: 1180, E: 0, B: 0): laranja 137, banana 136, maçã 125, abacaxi 103, uva 89, pera 82, mamão 69, manga 61, melancia 57 , abacate $40,(.$.

Instrumentos de limpeza (T: 39, C: 1123, E: 32, B: 25): vassoura 182, pano 131, rodo 130, detergente 77 , sabão 75 , balde 64 , água sanitária 53 , desinfetante 51 , escova 50 , palha de aço 46, ,...)

Instrumentos musicais (T: 37, C: 1147, E: 16, B: 16): violão 211, piano 134, guitarra 105, flauta 98, gaita, violino 87 , bateria 76 , pandeiro, saxofone 44 , cavaquinho $34,(.$.

Joias (T: 29, C: 1147, E: 14, B: 16): anel 211, brinco 186, pulseira 169, colar 139, relógio 101, corrente 83, aliança 66 , broche 31 , pingente 27 , gargantilha $25,(.$.

Meios de comunicação (T: 32, C: 1069, E: 48, B: 62): televisão 196, radio 184, telefone 167, internet 111, jornal 94, carta 83 , revista 50 , celular 42 , computador 39 , telegrama 28, (...)

Meios de transporte (T: 32, C: 1159, E: 6, B: 13): carro 213, ônibus 197, avião 160, trem 113, bicicleta 110 , moto 96 , metrô 67 , navio 47 , barco 29 , caminhão $21,(.$.

Móveis (T: 37, C: 1160, E: 17, B: 3): mesa 203, cadeira 174, cama 167, sofá 145, guarda-roupa 107 , armário 96 , estante 75 , cômoda 22 , raque 21 , banco $17,(.$.

Objetos associados ao esporte (T: 83, C: 1060, E: 73, B: 47): bola 218, tênis 107, raquete 106, rede 85 , chuteira 83 , meia 43 , uniforme 36 , apito 24 , camiseta 23 , bicicleta, trave $20,(\ldots)$

Objetos usados pela costureira (T: 24, C: 1148, E: 16, B: 16): linha 219, agulha 217, tesoura 175, máquina 150 , tecido 88 , fita métrica 75 , dedal 61 , alfinete 55 , botão 41 , régua $16,(.$.

Partes do corpo (T: 51, C: 1172, E: 3, B: 4): cabeça 179, braço 171, perna 166, mão 119, pé 115, olho 59, tronco 43 , pescoço 38 , boca 29 , nariz $23,(. .$.

Peças de vestuário (T: 44, C: 1170, E: 5, B: 5): calça 205, vestido 142, blusa 133, camisa 107, saia 100, meia 63 , calcinha 45 , casaco, cueca, sapato $41(.$.

Utensílios de cozinha (T: 76, C: 1160, E: 13, B: 5): panela 173, prato 143, faca 120, garfo 107, colher 104, copo 60 , talher 42 , frigideira 35 , fogão 33 , xícara $26,(. .$.

Nota: $\mathrm{T}=$ tamanho da categoria; $\mathrm{C}=$ número de respostas corretas; $\mathrm{E}=$ número de respostas incorretas; $\mathrm{E}=$ número de respostas em branco. 
Tabela 2. Normas de Categorias Semânticas de acordo com a Primeira Resposta Emitida

Animais (T: 24, C: 236, E: 0, B:0): cachorro 118, gato 41, cavalo 23, vaca 11, boi, leão 7, macaco 4, galinha, burro 3 , cabra, papagaio, porco, zebra $2,(.$.

Artigos escolares (T: 14, C: 235, E: 0, B: 1): caderno 83, lápis 70, livro 27, caneta 24, borracha 13, mochila 4, cola, régua 3 , papel, uniforme $2,(.$.

Artigos de higiene pessoal (T: 21, C: 236, E: 0, B: 0): sabonete 125, pasta de dente 31, escova de dente 22, escova 11, desodorante, papel higiênico 9, xampu 6, cotonete, lenço 3, creme, fio dental, perfume, sabão, toalha 2, (...)

Brinquedos (T: 21, C: 235, E: 1, B: 0): boneca 113, bola 48, carrinho 28, bicicleta 15, pião, velotrol 4, boneco, vídeo game 3, aviãozinho, balanço, barbie, bichinho de pelúcia 2, (...)

Coisas de bebê (T: 22, C: 232, E: 4, B: 0): fralda 78, chupeta 56, berço 21, mamadeira 19, roupa 10, macacão 6 , banheira, carrinho, chocalho 5, leite, sapatinho, talco 4, (...)

Coisas que servem para iluminar (T: 15, C: 231, E: 5, B: 0): lâmpada 134, lanterna 30, vela 29, luz 13, abajur, sol 5, lamparina 4, lampião 3, estrela 2, celular, farol, fogo, fósforo, lua, luminária 1

Doces (T: 41, C: 234, E: 2, B: 0): brigadeiro, de leite 33, bolo 27, chocolate 18, pudim 17, de goiaba 12, cocada 10, bala 9 , de abóbora 8 , pé de moleque $7,(. .$.

Ferramentas de carpinteiro (T: 29, C: 227, E: 6, B:3): martelo 99, serrote 53, alicate, enxada, plaina, serra 6, formão, pá 5 , machado 4, chave de fenda, enxó, esquadro, prego, serra elétrica, trena 3, (...)

Frutas (T: 26, C: 236, E: 0, B: 0): banana 55, maçã 47, laranja 44, manga 14, abacaxi 13, mamão 10, pera 9, abacate 6 , morango, uva $5,(\ldots)$

Instrumentos de limpeza (T: 19, C: 232, E: 4, B: 0): vassoura 108, sabão 29, pano 15, rodo 13, detergente 12, sabão em pó 11 , balde 9 , desinfetante 6 , bucha 5 , água sanitária, aspirador de pó $4,(.$.

Instrumentos musicais (T: 22, C: 232, E: 3, B: 0): violão 114, piano 38, gaita 18, flauta 15, guitarra 8, violino 7 , bateria 5, harpa, saxofone, tambor 4, (...)

Joias (T: 15, C: 234, E: 1, B:1): anel 112, brinco 31, relógio 25, colar 22, aliança 14, pulseira 10, corrente 7, ouro 3, argola, gargantilha, pérola $2,(.$.

Meios de comunicação (T: 10, C: 233, E: 2, B: 1): telefone 79, televisão 59, rádio 53, jornal 12, celular, internet 9 , computador 5 , carta 4 , revista 2 , e-mail 1

Meios de transporte (T: 16, C: 235, E: 1, B: 0): carro 103, ônibus 71, avião 19, bicicleta 10, trem 7, metrô 5, moto 4, caminhão, canoa, navio $3,(.$.

Móveis (T: 18, C: 234, E: 2, B:0): mesa 65, cama 45, cadeira 44, guarda-roupa 25, sofá 24, estante 8, armário 6 , televisão 4 , cômoda 3 , raque 2 , (...)

Objetos associados ao esporte (T: 18, C: 231, E: 4, B: 1): bola 156, tênis 25, chuteira 15, raquete 7, bicicleta, camisa, uniforme 4 , luva 3 , bola de futebol, calção, meia 2, (...)

Objetos usados pela costureira (T: 10, C: 235 , E: 1, B: 0): agulha 73, linha 62 , tesoura 43, máquina 37, tecido 8 , dedal 6 , alfinete 2 , fita métrica 2, bordado, carretel 1

Partes do corpo (T: 16, C: 236, E: 0, B: 0): cabeça 121, braço 34, mão 25, olho 16, perna 11, pé 9, boca, coração, nariz, orelha, rosto $3,(. .$.

Peças de vestuário (T: 21, C: 235, E: 1, B: 0): vestido 60, calça 50, camisa 44, blusa 28, calcinha 12, casaco 9, saia 6, cueca 5 , sapato 4 , sutiã $3,(\ldots)$

Utensilios de cozinha (T: 25, C: 233, E: 1, B: 1): panela 119, faca 31, prato 17, colher 16, garfo 11, fogão 7, caçarola, geladeira, talher 3, bacia, batedeira, caneca, concha, copo, escumadeira, panela de pressão 2, (...)

Nota: $\mathrm{T}=$ tamanho da categoria; $\mathrm{C}=$ número de respostas corretas; $\mathrm{E}=$ número de respostas incorretas; $\mathrm{E}=$ número de respostas em branco. 
Tabela 3. Frequência nas Regiōes Brasileiras dos Três Itens mais Comuns por Categoria

\begin{tabular}{|c|c|c|c|c|c|c|c|c|c|c|c|c|c|}
\hline \multirow{2}{*}{ Categorias } & \multicolumn{5}{|c|}{ região } & \multirow{2}{*}{ total } & \multirow{2}{*}{ Categorias } & \multicolumn{5}{|c|}{ região } & \multirow{2}{*}{ tota } \\
\hline & $\mathrm{CO}$ & $\mathrm{NE}$ & $\mathrm{N}$ & SE & $\mathrm{S}$ & & & $\mathrm{CO}$ & $\mathrm{NE}$ & $\mathrm{N}$ & SE & $\mathrm{S}$ & \\
\hline animais & \multicolumn{6}{|c|}{$f(\%) f(\%) f(\%) f(\%) f(\%) f(\%)$} & \multicolumn{7}{|c|}{ artigos higiene $f(\%) f(\%) f(\%) f(\%) f(\%) f(\%)$} \\
\hline cachorro & 19 & 17 & 18 & 18 & 15 & 17 & sabonete & 19 & 18 & 19 & 17 & 19 & 18 \\
\hline gato & 14 & 15 & 14 & 15 & 14 & 15 & xampu & 12 & 13 & 11 & 11 & 16 & 12 \\
\hline cavalo & 10 & 9 & 9 & 10 & 11 & 10 & pasta de dente & 15 & 11 & 13 & 11 & 11 & 12 \\
\hline \multicolumn{7}{|c|}{ artigos escolares } & \multicolumn{7}{|l|}{ brinquedos } \\
\hline lápis & 18 & 17 & 19 & 19 & 20 & 18 & boneca & 19 & 19 & 17 & 17 & 17 & 17 \\
\hline caderno & 18 & 16 & 16 & 18 & 20 & 17 & carrinho & 11 & 17 & 19 & 13 & 15 & 15 \\
\hline borracha & 15 & 16 & 7 & 15 & 12 & 14 & bola & 10 & 14 & 16 & 13 & 16 & 14 \\
\hline \multicolumn{7}{|c|}{ coisas de bebê } & \multicolumn{7}{|c|}{ coisas que servem para iluminar } \\
\hline fralda & 13 & 16 & 20 & 16 & 13 & 16 & lâmpada & 20 & 18 & 19 & 18 & 18 & 18 \\
\hline chupeta & 17 & 16 & 18 & 14 & 12 & 15 & lanterna & 18 & 15 & 17 & 17 & 18 & 16 \\
\hline mamadeira & 17 & 12 & 10 & 13 & 10 & 12 & vela & 12 & 14 & 17 & 13 & 16 & 14 \\
\hline \multicolumn{7}{|l|}{ frutas } & \multicolumn{7}{|c|}{ ferramentas de carpinteiro } \\
\hline laranja & 10 & 9 & 7 & 14 & 13 & 12 & martelo & 16 & 15 & 19 & 16 & 17 & 16 \\
\hline banana & 11 & 11 & 12 & 12 & 11 & 11 & serrote & 15 & 13 & 14 & 13 & 9 & 13 \\
\hline maçã & 13 & 12 & 7 & 11 & 8 & 11 & prego & 5 & 7 & 9 & 5 & 5 & 6 \\
\hline \multicolumn{7}{|c|}{ instrumentos musicais } & \multicolumn{7}{|l|}{ doces } \\
\hline violão & 19 & 17 & 18 & 18 & 17 & 18 & bolo & 1 & 7 & 16 & 6 & 10 & 7 \\
\hline piano & 17 & 11 & 12 & 11 & 10 & 11 & brigadeiro & 5 & 9 & 11 & 6 & 6 & 7 \\
\hline guitarra & 5 & 8 & 9 & 10 & 10 & 9 & de leite & 14 & 7 & 2 & 9 & 1 & 7 \\
\hline \multicolumn{7}{|l|}{ joias } & \multicolumn{7}{|c|}{ instrumentos de limpeza } \\
\hline anel & 19 & 18 & 19 & 18 & 18 & 18 & vassoura & 13 & 16 & 17 & 15 & 15 & 15 \\
\hline brinco & 19 & 16 & 17 & 16 & 16 & 16 & pano & 8 & 12 & 9 & 11 & 13 & 11 \\
\hline pulseira & 15 & 14 & 16 & 16 & 13 & 15 & rodo & 12 & 13 & 9 & 13 & 7 & 11 \\
\hline colar & 17 & 12 & 9 & 12 & 12 & 12 & detergente & 8 & 6 & 3 & 6 & 9 & 6 \\
\hline \multicolumn{7}{|c|}{ meios de transporte } & \multicolumn{7}{|c|}{ meios de comunicação } \\
\hline carro & 19 & 18 & 19 & 17 & 19 & 18 & televisão & 18 & 18 & 19 & 19 & 18 & 18 \\
\hline ônibus & 16 & 15 & 16 & 18 & 17 & 17 & rádio & 17 & 15 & 15 & 19 & 18 & 17 \\
\hline avião & 16 & 15 & 9 & 14 & 12 & 13 & telefone & 17 & 14 & 14 & 15 & 18 & 16 \\
\hline objetos usa & pela c & sture & & & & & móveis & & & & & & \\
\hline linha & 16 & 20 & 18 & 18 & 19 & 19 & mesa & 20 & 17 & 18 & 18 & 16 & 17 \\
\hline agulha & 17 & 19 & 19 & 18 & 18 & 18 & cadeira & 16 & 16 & 15 & 16 & 13 & 15 \\
\hline tesoura & 16 & 12 & 16 & 15 & 17 & 15 & cama & 17 & 15 & 16 & 15 & 11 & 14 \\
\hline partes do c & & & & & & & objetos associa & ao $\mathrm{c}$ & porte & & & & \\
\hline cabeça & 17 & 15 & 19 & 15 & 13 & 15 & bola & 20 & 19 & 17 & 19 & 17 & 18 \\
\hline braço & 16 & 13 & 14 & 15 & 14 & 14 & tênis & 13 & 7 & 9 & 9 & 10 & 9 \\
\hline perna & 17 & 13 & 13 & 15 & 13 & 14 & raquete & 10 & 10 & 7 & 8 & 10 & 9 \\
\hline utensílios d & zinha & & & & & & peças de vestua & & & & & & \\
\hline panela & 17 & 14 & 17 & 15 & 15 & 15 & calça & 18 & 16 & 17 & 19 & 17 & 17 \\
\hline prato & 14 & 11 & 13 & 12 & 13 & 12 & vestido & 13 & 13 & 15 & 10 & 13 & 12 \\
\hline faca & 4 & 11 & 10 & 11 & 10 & 10 & blusa & 13 & 14 & 12 & 11 & 8 & 11 \\
\hline garfo & 6 & 10 & 4 & 10 & 9 & 9 & camisa & 5 & 8 & 2 & 11 & 11 & 9 \\
\hline
\end{tabular}

Nota: $\mathrm{CO}=$ Centro-Oeste; $\mathrm{NE}=$ Nordeste; $\mathrm{N}=$ Norte; $\mathrm{SE}=$ Sudeste; $\mathrm{S}=\mathrm{Sul} ; \mathrm{f}(\%)=$ percentual de cada resposta na categoria. 
\& Bajo, 1988), essa forma de coleta gerou um maior número de conceitos por categoria, ou seja, aumentou o set size.

$\mathrm{Na}$ análise da primeira resposta emitida, o tamanho da categoria varia de 10 a 41 conceitos por categoria. Esse número aumenta para 24 a 109 conceitos por categoria quando levado em consideração as cinco respostas emitidas pelos participantes. É importante salientar que, ao coletar somente um item, perde-se a oportunidade de compreender se há exemplares tão prototípicos ou representativos quanto o primeiro recordado, que competem com este em sua frequência. O set siže aumentado propicia a compreensão de itens menos prototípicos em maior escala, tendo em vista que, na solicitação de geração, somente do primeiro exemplar, a probabilidade de itens menos prototípicos aparecerem é menor. À medida que o número de conceitos por categoria aumenta, entende-se que há uma perda na força de associação semântica deles, por diminuir a frequência de respostas de cada conceito associado à categoria.

Em comparação aos outros bancos de dados, percebe-se que há uma similaridade no que diz respeito aos itens mais frequentes quando avaliadas as mesmas categorias semânticas. Por exemplo, na categoria brinquedos (primeira resposta emitida), no presente estudo, obteve-se, em ordem com maior frequência, "boneca, bola e carro". Tais achados também foram encontrados nos estudos de Janczura (1996) e Battig e Montague (1969). Van Erven (2004) encontrou resultados parecidos, (ordem de frequência: boneca, carro e bola), com pouca diferença na frequência de respostas para os itens "bola" e "carro", mostrando uma similaridade, mesmo em culturas distintas.

Embora haja bastante similaridade dos resultados entre os estudos, alguns itens como, por exemplo, "meia" para artigos de vestuário, apresentou maior frequência no estudo internacional de Battig e Montague (1969) - segundo item mais frequente; e nos estudos brasileiros, tem frequência reduzida. Outro exemplo é o item "luva" da mesma categoria, que, nos estudos brasileiros, apresenta frequência igual a 1 , tanto no estudo presente como no de Janczura (1996), não foi listado no estudo de Van Erven (2004), e apresenta maior frequência no estudo internacional (Battig \& Montague, 1969). Tais achados devem estar associados a questões climáticas dos locais no qual as coletas foram realizadas e reforçam a importância de normas de categorização semânticas brasileiras.

Os resultados apontaram que os conceitos mais frequentes nas categorias, também, o foram quando consideradas sua frequência por região brasileira.
Porém, destaca-se que alguns conceitos, como, por exemplo, "metrô", não foi emitido pelos participantes de todas as regiões. Esse dado reforça o cuidado dos pesquisadores na utilização dessas normas, levando em consideração a diversidade cultural brasileira para maior validade na interpretação dos achados de pesquisa.

Da mesma forma, faz-se necessário a devida atenção e cuidado na escolha dos exemplares das categorias, como itens de instrumentos e tarefas de avaliação, tendo em vista que itens mais frequentes são os que apresentam maior rapidez de acesso como pertencentes à categoria (Janczura \& Nelson, 2006). Tendo em vista o objetivo do teste, como é o caso de testes de habilidades, pode-se optar pela seleção de itens com diferentes gradações de dificuldades, ou seja, poder-se-ia pensar em itens com diferentes forças de associação com a categoria (Koriat, 200), porém, sem deixar de levar em conta a presença deles em todas as regiões. Esse cuidado tem sua importância principal na validade da avaliação, tendo em vista que o desconhecimento em função da não familiaridade de um conceito não pode ser julgado como deficiência cognitiva.

Por fim, no que diz respeito às respostas dos participantes, o número considerado como erros e em branco foi menor nesse estudo (análise do primeiro item), comparado com de Janczura (1996). Battig e Montague (1969) não realizaram essa avaliação, listando todas as respostas dadas pelos participantes. Um dos motivos pode ter sido a decisão dos autores desse artigo em manter alguns itens como pertencentes a uma determinada categoria, apesar de conceitualmente não o serem. Optou-se por validar o conhecimento tácito dos participantes relativo a categorias que apresentaram frequência elevada em conceitos que categoricamente estariam incorretos. Assim, por exemplo, foram considerados como pertencentes à categoria ferramentas de carpinteiro os exemplares "formão" e "betoneira", o que de fato faz parte dos instrumentos para a construção. Porém, quando se considera as cinco respostas emitidas, o número de respostas consideradas como "em branco", nesse estudo, é superior ao estudo de Janczura (1996). Esse resultado faz sentido, tendo em vista a maior dificuldade de emitir cinco conceitos de cada categoria do que somente um, e a cada conceito apresentado reduz-se um do universo possível de alternativas dentro da categoria semântica.

\section{Considerações Finais}

O estudo apresenta normas brasileiras de associação para 20 categorias semânticas e tem a pretensão 
de fornecer contribuições a pesquisadores que atuam, principalmente, na área de neuropsicologia e na construção de instrumentos de avaliação cognitiva, como testes de memória, aprendizagem, linguagem, entre outros. Destaca-se a preocupação do presente estudo com a representatividade das cinco regiões brasileiras, buscando-se fornecer dados mais confiáveis quando de sua generalização para o Brasil.

A amostra reduzida (embora estratificada) impossibilitou algumas análises mais robustas. Estudos futuros com a ampliação da amostra possibilitariam análises quantitativas de padrões de categorização por região, idades e escolaridade.

De modo geral, os resultados deste estudo estão de acordo com a literatura e bancos de dados brasileiros sobre a temática (Janczura, 1996; Van Erven, 2007). Por meio da coleta de dados em todas as regiões brasileiras, este estudo amplia o conhecimento de como os brasileiros acima de 40 realizam associações dentro das categorias semânticas, quais são os conceitos mais prototípicos por categoria em cada região, além de apresentar conceitos comuns às regiões.

\section{Referências}

Battig, W., \& Montague, W. E. (1969). Category norms for verbal items in 56 categories: a replication and extension of the connecticut category norms. Journal of Experimental Psychology Monograph, 80(3), 1-24.

Buschke, H. (1984). Cued recall in amnesia. Journal of Clinical and Experimental Neuropsychology, 6, 433-440.

Cohen, B. H., Bousfield, W. A., \& Whitmarsh, G. A. (1957). Cultural norms for verbal items in $43 \mathrm{ca}$ tegories. Connecticut Univ Storrs: University of Connecticut.

Hampton J. A. \& Gardiner, M. M. (1983). Measures of internal category structure: A correlational analysis of normative data. British JournalofPsychology, 74(4), 491-516.

Instituto Brasileiro de Geografia e Estatística (2011). Censo 2010. Disponível em http://www.ibge.gov.br/

Janczura, G. A. (1996). Normas associativas para 69 categorias semânticas. Psicologia: teoria e pesquisa, 12(3), 237-244.

Janczura, G. A. (2005). Contexto e normas de associação para palavras: A redução do campo semântico. Paideia, 15(32), 417-435.
Janczura, G. A., Castilho, G. M., Rocha, N. O., Van Erven, T. J. C., \& Huang, T. P. (2007). Normas de concretude para 909 palavras da língua portuguesa. Psicologia: teoria e pesquisa, 23(2), 195-204.

Janczura, G. A., \& Nelson, D. L. (2006). A hipótese da acessibilidade conceitual para a tipicidade e a produção linguística. Psicologia: reflexão e crítica, 19(3), 491-497.

Kaplan, E., Goodglass, H., \& Weintraub, S. (2001). Boston naming test, second edition. Philadelphia: Lippincott Williams \& Wilkins.

Koriat, A. (2000) Control processes in remembering. Em E. Tulving,\& F. I. M. Craik. The handbook of memory. Oxford University Press, New York.

Kucera, H., \& Francis, W. (1967). Computational analysis of present-day American English. Providence, RI: Brown University Press.

McEvoy, C. L., \& Nelson, D. L. (1982). Category name and instance norms for 106 categories of various sizes. American Journal of Psychology, 95, 581-634.

Nelson, D. L., McEvoy, C., \& Bajo, M. T. (1988). Lexical and semantic search in cued recall, fragment completion, perceptual identification, and recognition. American Journal of Psychology, 101(4), 465-480.

Paivio, A., Yuille, J. C., \& Madigan, S. A. (1968). Concreteness, imagery, and meaningfulness values for 925 nouns. Journal of Experimental Psychology, 76(1), 1-25.

Petersen, R.C., Smith, G., Kokmen, E., Ivnik, R. J., \& Talangos, E.G. (1992). Memory function in normal aging. Neurology, 42, 396-401.

Robson, C. (1993). Real world research: A resource for social scientists and practitioner-researchers. Oxford: Blackwell Publishers.

Salles, J. F., Holderbaum, C. S., Becker, N., Rodrigues, J. C., Liedtke, F. V., Zibetti, M. R., \& Piccoli, L. F. (2008). Normas de associação semântica para 88 palavras do português brasileiro. Psico, 39(3), 260-268.

Sardinha, T. B. (2000). Linguística de corpus: Histórico e problemática. Documentação de Estudos em Linguistica Teórica e Aplicada, 16(2), 323-367.

Snodgrass, J. G., \& Vanderwart, M. (1980). A standardized set of 260 pictures: Norms for name agreement, image agreement, familiarity, and visual 
complexity. Journal of Experimental Psychology: Human Learning and Memory, 6(2), 174-215.

Stein, L. M., Feix, L. F., \& Rohenkohl, G. (2006). Avanços metodológicos no estudo das falsas memórias: Construção e normatização do procedimento de palavras associadas. Psicologia Reflexão e Crítica, 19(2), 166-176.

Stein, L. M. \& Gomes, C. F. A. (2009). Normas brasileiras para listas de palavras associadas: Associação semântica, concretude, frequência e emocionalidade. Psicologia: teoria e pesquisa, 25(4), 537-546.

Van Erven, T. J. C. G. (2010) A Base de Classificação Categórica em Idosos. Unplublished masther's thesis, Programa de Pós-Graduação em Ciência do Comportamento, Instituto de Psicologia, Universidade Federal de Brasília. Brasília, Brasil.
Van Erven, T. J. C. G., \& Janczura, G. A. (2007). Normas associativas para categorias semânticas em idosos (Pesquisa em curso). Universidade de Brasília. Brasília, DF.

Zortea, M., \& Salles, J. F. (2012). Estudo comparativo das associações semânticas de palavras. Psicologia: teoria e pesquisa, 28(3), 259-266.

Yoon, C., Feinberg, F., Gutchess, A. H., Hedden, T., Chen, H. M., Hu, P.,... Park, D. C. (2004). Category norms as a function of culture and age: Comparisons of item responses to 105 categories by American and Chinese adults. Psychology and Aging, 19(3), 379-394.

Recebido em: 09/05/2014 Primeira Reformulação em: 27/06/2014

Aprovado em: 16/01/2015 
Nota dos autores:

Agradecimento especial à Dra. Terezinha de Jesus Cordeiro van Erven pela colaboração e disposição de seu material de normas de categorias semânticas para idosos.

Este estudo recebeu apoio do CNPq para sua realização.

Sobre os autores:

Suelen Bordignon é psicóloga, especialista em Terapia Cognitivo-Comportamental, doutoranda e mestre em Psicologia pela Universidade Federal do Rio Grande do Sul (UFRGS), e integrante do Núcleo de Estudos em Avaliação Psicológica e Psicopatologia (NEAPP).

Murilo Ricardo Zibetti é psicólogo graduado na UFRGS, com experiência clínica e de ensino em Avaliação Neuropsicológica e Reabilitação Neuropsicológica, mestre e doutorando em Psicologia, com ênfase em avaliação psicológica no programa de pós-graduação em Psicologia na Universidade Federal do Rio Grande do Sul (UFRGS).

Clarissa Marceli Trentini é professora associada dos cursos de graduação e pós-graduação em Psicologia do Instituto de Psicologia - UFRGS, coordenadora do Núcleo de Estudos em Avaliação Psicológica e Psicopatologia (NEAPP), doutora em Ciências Médicas: Psiquiatria - UFRGS, e bolsista CNPq.

Ana Cristina Resende é professora adjunta da PUC Goiás, pós-doutoranda na UNIFESP, com bolsa da FAPESP, doutora em Psicologia pela PUC-RS (2009), com estágio de doutorado pela California School of Professional Psychology at Alliant International University - San Diego, CA, e diretora do IGAP.

Carla Alexandra da Silva Moita Minervino é doutora em Ciências da Saúde pela UFPB (2004), professora da UFPB, líder do Núcleo de Estudos em Saúde Mental, Educação e Psicometria (NESMEP), professora da pós-graduação em Neurociência Cognitiva e Comportamento, e realizou estágio pós-doutoral em Psicologia Cognitiva na UFPE.

José Humberto da Silva Filho é doutor em Psicologia - USP, professor adjunto na graduação e pós-graduação em Psicologia - UFAM, neuropsicólogo, fundador e coordenador do Laboratório de Avaliação Psicológica do Amazonas na UFAM (LAP-AM) e do Laboratório de Medidas e Avaliação Psicológica na Faculdade Martha Falcão (LAMAP-FMF).

Josiane Pawlowski é psicóloga, doutora em Psicologia pela Universidade Federal do Rio Grande do Sul (UFRGS) e professora adjunta do departamento de Psicometria do Instituto de Psicologia da Universidade Federal do Rio de Janeiro (UFRJ).

Maycoln L. M. Teodoro é professor adjunto do programa de pós-graduação em Psicologia da Universidade Federal de Minas Gerais, doutor pela Albert-Ludwigs-Universität Freiburg (Alemanha), com estágio pós-doutoral na UFRGS, e bolsista produtividade do CNPq.

Neander Abreu é psicólogo, especialista em Neuropsicologia, professor adjunto do Instituto de Psicologia da Universidade Federal da Bahia, doutor em Psicologia (Neurociências e Comportamento, 2007) pela Universidade de São Paulo, pós-doutor com bolsa CAPES na Université du Luxembourg e The University of York (2013).

Contato com os autores:

Suelen Bordignon

Rua Antônio Michelon, 498. Santa Rita, Bento Gonçalves, RS

CEP: 95700-000

E-mail: bordignon.suelen@gmail.com 